\title{
Studying of the dielectric loss factor of grain by indirect method
}

\author{
Dmitry Budnikov ${ }^{1,}$, Alexey Vasilev ${ }^{1}$, and Alexey Vasilev ${ }^{1}$ \\ ${ }^{1}$ Ministry of Science and Higher Education of the Russian Federation, Federal State Budgetary \\ Scientific Institution "Federal Scientific Agroengineering Center VIM" (FSAC VIM), 109428, $1^{\text {st }}$ \\ Institutsky, 5, Moscow, Russia
}

\begin{abstract}
Significant losses of grain crops at various stages of cultivation, harvesting, post-harvest processing and storage dictate the need to technologies and equipment for these processes. Currently, one of the most promising directions in the field of heat treatment of grain is the use of combined influence methods with the use of electrophysical effects. The purpose of this work is to develop and test a method for measuring the dielectric loss factor by an indirect method, which makes it possible to control the humidity of grain material exposed to microwave. The paper presents the equipment and the design of the experimental installation. The results of the experiment were subjected to regression analysis. The results of experimental studies and dependencies can be used for modelling microwave effects on agricultural bulk materials in electromagnetic modelling software, such as CST Studio.
\end{abstract}

\section{Introduction}

The study of combined impact methods on agricultural materials and the development of energy-efficient equipment is largely associated with the development of industrial equipment based on mathematical and computer models. Wide opportunities for the development of such equipment are provided by the use of specialized software tools. As a result, the use of microwave fields will reduce the cost of heat treatment by $15-20 \%$, depending on the process and the type of material being processed [1-4]. To provide the required parameters applicable to the drying equipment, it is necessary to provide uniform heating of the processed material by the volume of the pipeline. Modern means of computer modelling allow for prototyping and visual representation of the treatment area of the material [5-7]. For modelling and further development prior to con-ducting mathematical modelling and sample making equipment, it is necessary to investigate the properties of crops.

Dielectrics are a class of materials that are poor conductors of electricity, as op-posed to materials such as metals, which are generally good electrical conductors. Many materials, including food, living organisms, and most agricultural products, conduct electric current to some extent, but they are still classified as dielectrics. The electrical nature of these

\footnotetext{
* Corresponding author: Dimm13@inbox.ru
} 
materials can be described by their dielectric properties, which affect the distribution of electromagnetic fields and currents in the area occupied by the materials, and which determine the conductivity of materials in electric fields. Thus, the dielectric properties determine how quickly the material will heat up in the RF or microwave heating field. Their influence on electric fields also makes it possible to create means of measuring certain other properties of materials that can be correlated with dielectric properties through non-destructive electrical measurements. The dielectric properties of agricultural products are important for determining the quality of measurements in the agricultural industry, as well as in the field of dielectric heating.

Simplified definitions of dielectric properties are useful when discussing their application. The main characteristic of all forms of electromagnetic energy is their propagation through free space at the speed of light $c$. The speed of propagation of electromagnetic energy in a material $v$ depends on the electromagnetic characteristics of this material and is defined as:

$$
v=\frac{1}{\sqrt{\mu \cdot \varepsilon}}
$$

where $\mu$ - magnetic permeability of the material; - dielectric constant.

The absolute permittivity $\varepsilon$ can be represented as a complex value:

$$
\varepsilon=\varepsilon^{\prime}-j \varepsilon^{\prime \prime}
$$

where $\varepsilon^{\prime}-$ real part, called dielectric constant; $\varepsilon^{\prime \prime}$ - imaginary part, called dielectric loss factor.

These values are dielectric properties of practical interest. Dielectric constant $\varepsilon^{\prime}$ associated with the ability of a material to store energy of electric field in the material and loss factor $\varepsilon^{\prime \prime}$ is related to the ability of a material to absorb or dissipate energy, in other words to convert electrical energy into thermal energy. The dielectric loss coefficient is an indicator of the ability of a material to heat in a microwave field. Permittivity is also important because of its effect on the distribution of electric fields. For example, the electrical capacity of two parallel conducting plates separated by free space or air will be multiplied by the permittivity of the material if the space between the plates is filled with this material.

At various times, scientists around the world have been researching the dielectric properties of agricultural products [1], [7-9]. Based on these data, it can be concluded that the dielectric properties of cereals significantly depend on humidity, frequency and temperature, while even under the same conditions within the same crop, the data may differ significantly, which can be associated with the characteristics of the variety. In addition, all data are given for a dense layer, since determining the dielectric proper-ties is associated with a number of technical difficulties.

The most detailed studies of the dielectric properties of cereals are presented in the works of S. O. Nelson [8]. His work presents the properties of both grain crops, such as wheat, barley, rice, etc., and grain pests. These dependencies are represented by temperature, humidity of the material, and frequency of the exposure field.

In these studies, there is a significant difference in the dependence of the dielectric properties of grain products and pests, which is used for pest control when processing products during storage [10-15]. 


\section{Materials and methods}

Taking into account the fact that the permittivity is related to a number of other values (electric field strength, capacity, material type, etc.), the measurements are indirect. The idea is based on experimental obtaining of measured parameters in various modes, which can be used to calculate $\varepsilon$. In this case, the means and methods of measurement depend on the appropriate types of crops under study, the frequency of the affected electromagnetic radiation, the required measurement accuracy, etc.

For the frequency range below $200 \mathrm{MHz}$, bridge resistor circuits and resonant circuits are used. At frequencies above $200 \mathrm{MHz}$, transmission lines, resonant technology, waveguide and coaxial systems are used.

According to Russian Standard GOST R 8.623-2006, the dielectric permittivity and the tangent of the dielectric loss angle of solid dielectrics in the frequency range from 1 to 20 $\mathrm{GHz}$ is measured using a metal-dielectric resonator.

\subsection{Existing methods for determining dielectric properties}

Electrical insulation materials, which include grain, can have different types of polarization: electronic, ionic, migration, spontaneous, elastic. Each of them has its own frequency limit. Therefore, to measure the relative permittivity $\varepsilon$ and the tangent of the dielectric loss angle $\operatorname{tg} \delta$, it is necessary to use frequency-appropriate methods.

Grain of various agricultural crops is a typical dielectric. Measurement of the dielectric properties of substances is based on the reflection or passage of electromagnetic radiation through the substance, using resonant or non-resonant systems with an open or closed structure for experimental determination of the parameters of the samples under study. Waveguides or coaxial transmission lines are closed structures, while measurements of open propagation of electromagnetic radiation and open-closed coaxial lines are open systems.

Since the resonant frequency and q-factor of oscillating systems can be measured with high enough accuracy, resonant methods for measuring the parameters of matter are the most perfect. At moderately high frequencies up to $300 \mathrm{MHz}$, q-meters or full conductivity meters are usually used as oscillating systems. For measurements, a special sample capacitor is used, between the plates of which a plate of the investigated dielectric is placed. This capacitor is connected in parallel to the q-meter circuit.

The method of measuring the parameters of the dielectric is as follows. With the use of a Q-meter, the capacitance of a sample capacitor is measured with and without a sample of the studied dielectric.

The methods presented above have their advantages and disadvantages, and the feasibility of using each of them is determined by the conditions of the experiment. The main difficulty lies in the fact that the most accurate methods for the microwave range require that the object under study is a monolithic sample with certain geometric parameters, only then will the measurement accuracy be achieved, which is not quite convenient for cereals due to the size, the resonant methods remain the most correct at the moment.

\subsection{The proposed methods for indirect determining dielectric loss factor}

In cases where it is necessary to determine the humidity of the material in continuous mode, for example, in grain heat treatment plants for continuous monitoring of the humidity of the processed material, such methods are not suitable. In addition, they are not applicable for a 
loose layer of material. Thus, it is necessary to develop an alternative method that has high losses, but can be used in technological processes.

The proposed method was developed to study the state of grain material subjected to the combined effect of microwave energy $(2.45 \mathrm{GHz})$ and a drying agent.

\subsubsection{Control and measuring devices}

As mentioned earlier, the existing methods for measuring dielectric properties are indirect and are designed to measure the parameters of the material directly. In the case of grain heat treatment using electromagnetic fields, we are dealing with a grain-air mixture. In this case, the grain layer can be in various states (dense stationary, dense mobile, fluidized, suspended).

From the point of view of development and modelling of grain drying equipment in which the microwave field is used, we need to know exactly the properties of the corresponding grain layer.

Since the determination of the specific power released in the grain layer re-quires knowledge of the dielectric loss coefficient of the material, the developed method will be aimed at determining this characteristic. As temperature sensors, both thermocouples and optical temperature sensors were used to exclude the influence of electromagnetic fields on the measuring system during the influence of the microwave field.

The following equipment was used for measurements:for process control and registration of optical signals from sensors were used unified recording module (URM) FIU-44-1.55-40-S-A4-IP40-2;fiber-optic temperature sensors OSMT-313; thermal anemometer TTM-2-02-2;moisture meter Fauna-M.

The laboratory setup shown in figure 1 allows studying the distribution of the microwave field strength in a dense grain layer.

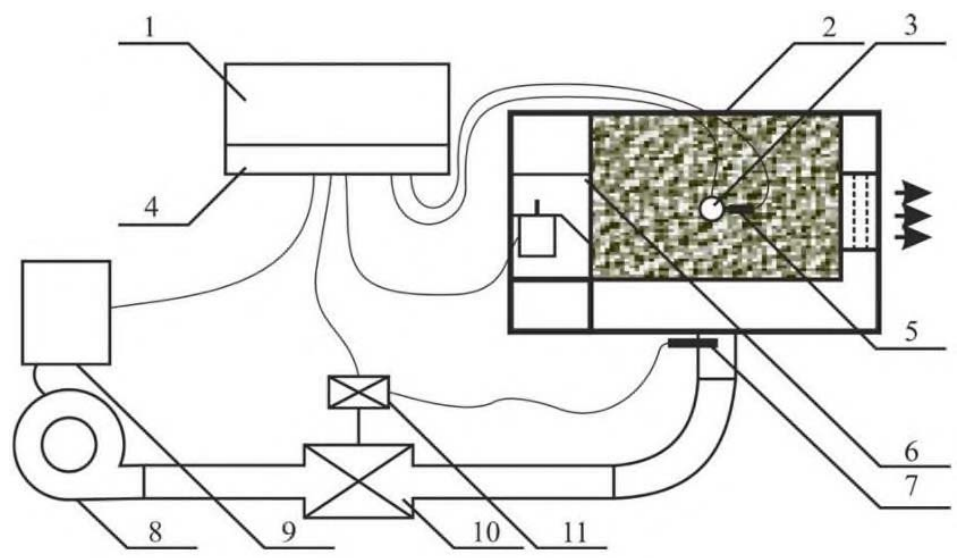

Fig. 1. Laboratory installation of a dense layer: 1 - PC, 2 - microwave convection processing zone, 3 calorimetric sensor, 4 - input / output board, 5 - grain layer temperature sensor, 6 - microwave source (magnetron, power supply, waveguide, 7 - air temperature sensor, 8 - fan, 9 - vector frequency converter, 10 - heater, 11 - thermoreactor

Knowing the field strength at a specific point, and measuring the temperature change during the control, the dielectric loss factor can be determined analytically by the dependence:

$$
\varepsilon^{\prime \prime}=\frac{c_{m} \cdot \rho_{m} \cdot \Delta T}{5.56 \cdot 10^{-11} \cdot E^{2} \cdot f \cdot \tau}
$$


where $c_{m}$ - heat capacity of the material, $\mathrm{kJ} / \mathrm{kg} \cdot{ }^{\circ} \mathrm{C} ; \rho_{m}$ - dry substance density of the sample, $\mathrm{kg} / \mathrm{m}^{3} ; \Delta T$ - heating of the material during measurement, ${ }^{\circ} \mathrm{C} ; E$ - electric field strength, $\mathrm{V} / \mathrm{m} ; f$ - frequency of the electromagnetic field, Hz.

\subsubsection{Method of indirect determination of the dielectric losses coefficient of the grain layer}

For measurements using this method in the frequency range of $0.4-2.5 \mathrm{GHz}$, there are used samples that fill the zone of microwave convective action, with a relative permittivity from 2 to 100 . A special feature of the method is filling the measurement zone with grain with the desired layer density.

In the proposed method, samples that fill the zone of microwave convective action with a relative permittivity from 2 to 100 are used for measurements. A schematic representation of the laboratory installation for measurements is shown in figures 1 (for the dense layer) and 2 (for the fluidized and suspended layers).

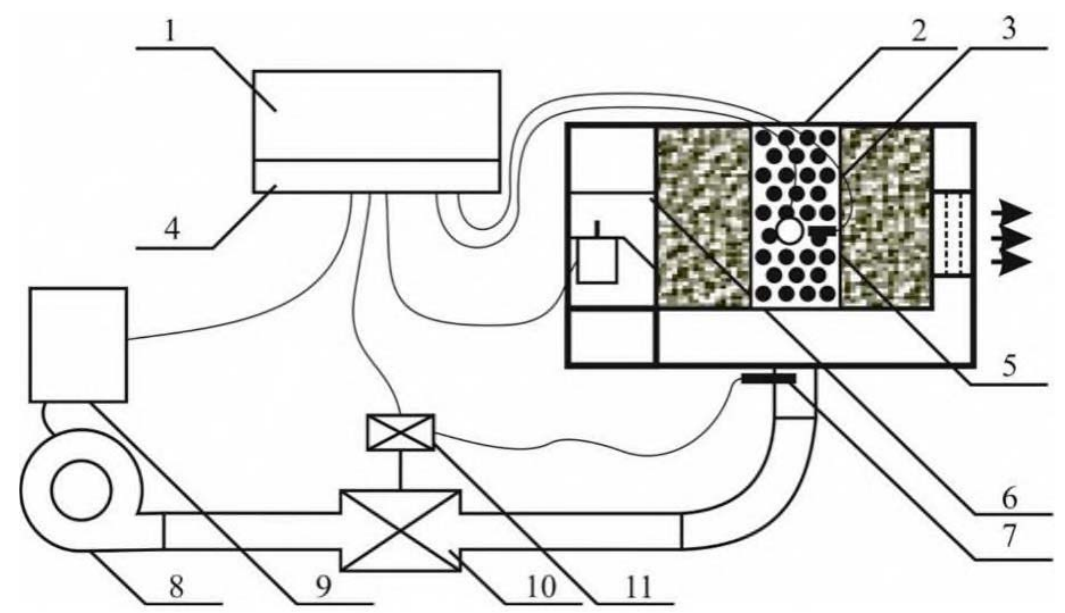

Fig. 2. Laboratory installation of a suspended layer: 1 - PC, 2 - microwave convection processing zone, 3 - calorimetric sensor, 4 - input / output board, 5 - grain layer temperature sensor, 6 microwave source (magnetron, power supply, waveguide, 7 - air temperature sensor, 8 - fan, 9 vector frequency converter, 10 - heater, 11 - thermoreactor

Studying of relative permittivity consists in measuring the heating temperature of the grain layer (grain) in the zone of microwave convective processing with known values of the field strength at the control point, the dependence of the heat capacity of the material under study on its temperature and humidity. Fiber-optic temperature sensors can be used to increase the accuracy of measurement in a dense layer.

Preparing to perform measurements. When preparing to perform measurements, the following works are performed:

- assemble the measuring unit according to the scheme shown in figure 2 (for a dense layer) or 3 (for fluidized and suspended layers);

- all devices included in the measuring unit are prepared for operation in accordance with their technical documentation, including the launch of the SCADA measurement control system;

- samples are prepared for measurements in accordance with the appropriate technological process, for which the obtained characteristics will be used in the future. At the same time, 
it is necessary to check the absence (presence) of impurities, uniform humidity by volume, dustiness, etc.

Performing measurements. When performing measurements of the dielectric loss coefficient by the pro-posed method, the following operations are performed:

For a dense layer:

- temperature changes are monitored during 3 heating periods ( 5 seconds of microwave heating, 15 seconds for temperature distributing by grain volume);

- temperature growth is controlled by thermocouples in the center and on the surface of the grain, as well as by a fiber-optic temperature sensor on the surface (in the layer).

For fluidized and suspended layer:

- temperature changes are monitored during 3 heating periods (5 seconds of microwave heating, 15 seconds for temperature distribution into grain volume);

- temperature growth is controlled by thermocouples in the center and on the surface of the grain, as well as by a fiber-optic temperature sensor on the surface (in the layer).

\section{Results and discussions}

The coefficient of dielectric loss of the material under study in a dense layer is calculated from the equation (3).

The dielectric loss factor of the material under study in the fluidized and suspended layer (during ventilation) is calculated from the equation:

$$
\varepsilon^{\prime \prime}=\frac{c_{m} \cdot \rho_{m} \cdot \Delta T+K \cdot\left(T-T_{0}\right)}{5.56 \cdot 10^{-11 \cdot E^{2} \cdot f \cdot \tau}}
$$

where $T$ and $T_{0}$ - load and ambient temperatures, ${ }^{\circ} \mathrm{C} ; K$ - coefficient of thermal scattering, $\mathrm{J} / \mathrm{s} \cdot{ }^{\circ} \mathrm{C}$.

When determining the coefficient of dielectric loss of samples of the studied material in the dense layer, the values obtained by the fiber-optic sensor and the aver-age value between the temperature of the surface and the center of the grain are calculated in parallel.

When determining the coefficient of dielectric loss of samples of the material under study in a fluidized and suspended layer (during ventilation), calculations are made on the average value between the temperatures of surface and center of the grain.

When determining the coefficient of dielectric loss of samples of the material under study, at least five measurements are made and the result is taken as the arithmetic mean.

Figure 3 shows an example of the obtained graphs for determining the coefficient of dielectric loss for wheat at humidity from 11 to $30 \%$ for three levels of material density.

The results obtained for different densities of the material correspond to the general dynamics, but differ significantly in level. For a dense layer, which corresponds to the density of $800 \mathrm{~kg} / \mathrm{m}^{3}$ in the range of moisture of $11 \div 30 \%$, the coefficient of dielectric loss ranges from 0.18 to 0.42 ; for fluidized layer $\left(500 \mathrm{~kg} / \mathrm{m}^{3}\right)$ it is from 0.06 to 0.15 ; for weighted one $\left(200 \mathrm{~kg} / \mathrm{m}^{3}\right)$ it is from 0.1 to 0.3 . The accuracy of the data for different density levels differs significantly as in the dense layer the error is caused by the used sensors, as well as the presence of air gaps in the layer; for fluidized and suspended layers, in addition to the specified losses are added due to the thermal interaction of air with the grain layer and the sensor.

The results of experiments performed for some grain materials were processed using Matlab and presented as a polynomial model:

$$
\varepsilon^{\prime \prime}=a_{0}+a_{1} \cdot W+a_{2} \cdot \rho+a_{3} \cdot W^{2}+a_{4} \cdot W \cdot \rho+a_{5} \cdot \rho^{2},
$$

where $a_{i}$ - model proportionality coefficients; $\rho$-density of the processed material, $\mathrm{kg} / \mathrm{m}^{3}$. 
Results are presented in table 1.

Table 1. Formatting sections, subsections and paragraphs.

\begin{tabular}{|c|c|c|c|c|c|c|c|c|c|}
\hline Material & $a_{0}$ & $a_{1}$ & $a_{2}$ & $a_{3}$ & $a_{4}$ & $a_{5}$ & $\mathrm{SSE}$ & $\mathrm{R}^{2}$ & $\mathrm{RMSE}$ \\
\hline Wheat & 0.4232 & 0.3525 & 1.042 & $0 ., 03711$ & 0.3813 & 0.7108 & 0.6609 & 0.9549 & 0.2710 \\
\hline Barley & 0.562 & 0.3329 & 0.08991 & 0.06301 & 0.05348 & -0.0114 & 0.0024 & 0.9987 & 0.0164 \\
\hline Oats & 0.5041 & 0.2005 & 0.001605 & 0.03722 & -0.0528 & 0.03904 & 0.07621 & 0.8968 & 0.0920 \\
\hline Tritikale & 0.5852 & 0.3525 & 0.6362 & -0.003194 & 0.2487 & 0.3868 & 0.1801 & 0.9808 & 0.1414 \\
\hline
\end{tabular}

\section{Conclusion}

1 The proposed method can be used in the development of equipment for monitoring the state of the grain layer in grain heat treatment plants with combined microwave-convective action.

2 The dependence of the dielectric loss factor of agricultural grain materials on humidity and layer density can be represented by a complete second-order polynomial dependence.

3 It is possible to increase the accuracy of measurements and the quality of the obtained regression models by using optical temperature sensors.

4 Further research on the properties of grain materials is required to in-crease the range of data on the dielectric properties of both different crops and different varieties.

5 The obtained results of experimental studies and dependencies can be used for modelling microwave effects on agricultural bulk materials in electromagnetic modelling software, such as CST Studio.

\section{References}

1. Y. Zhao, W. Wang, et al. Drying Technol, 35(13), 1561-1570 (2017)

2. D. A. Budnikov, The Use of Microwave Energy at Thermal Treatment of Grain Crops. Handbook of Research on Renewable Energy and Electric Resourcesfor Sustainable Rural Development, 475-499 (USA, PA, Hershey: IGI Global, 2018) ISBN 9781522538677 DOI: 10.4018/978-1-5225-3867-7.ch020

3. De Freitas et al. Degradation kinetics of pirimiphos-methyl residues in maize grains exposed to ozone gas. Journal of stored products research, 74(1), 1-5 (2017) DOI: 10.1016/j.jspr.2017.08.008

4. A. Yu. Izmailov, Herald of the Russian academy of sciences, 89(2), 209-210 (2019) DOI: $10.1134 / \mathrm{S} 1019331619020072$

5. A. N. Vasiliev, V. P. Goryachkina, B. Dmitry, International Journal of Energy Optimization and Engineering (IJEOE), 9(2), , 11 (2020) DOI: 10.4018/IJEOE.2020040101

6. D. A. Budnikov, A. N. Vasiliev, Intelligent Computing \& Optimization, 866, 139-145 (2018) DOI: 10.1007/978-3-030-00979-3_14

7. M. Fazaeli, S. Yousefi, Z. Emam-Djomeh, Food Res Int. 50(2), 568-573 (2013)

8. S. O. Nelson, Dielectric Properties of Agricultural Materials and Their Applications. Academic Press, 229 (2015) 
9. B. Ling, J. G. Lyng, S. Wang, Innovative Food Science \& Emerging Technologies, 48, 66-74 (2018) DOI: 10.1016/j.ifset.2018.05.012

10. T. Basak, M. Bhattacharya, S. A. Panda, Innovative Food Sci Emerg Technol, 33, 333-347 (2016)

11. W. Guo, S. Wang, G. Tiwari, J. A. Johnson, J. Tang, LWT-Food Sci Technol, 43(2), 193-201 (2010)

12. A. B. Ospanov, A. N. Vasilev, et al., J.Eng. Applied Sci., 11 (Special Issue 1), 29152919 (2016)

13. M. Ranjbaran, D. Zare, C. Energy, 59, 484-493 (2013) DOI: dx.doi.org/10.1016/j.energy.2013.06.057

14. A. N. Vasiliev, A. B. Ospanov, D. A. Budnikov et al., Improvement of grain drying and disinfection process in the microwave field. Monography, Almaty: Nur-Print, 155 (2017) ISBN 978-601-7869-72-4

15. A. N. Vasiliev, D. A. Budnikov, Advances in Intelligent Systems and Computing book series (AISC), 866, 239-245 (2019) DOI: 10.1007/978-3-030-00979-3_24 